\title{
Correspondence
}

\section{Anaesthetic drug costs are not increased by propofol}

To the Editor:

The relatively high cost of propofol has prohibited its introduction to many institutions. At our institution, we can determine the exact quantities of drugs used in any month in the Operating Room. Propofol was introduced on an interim basis in December, 1990, with its use restricted to outpatients. A breakdown of the costs is as follows:

\begin{tabular}{ll}
\hline Monsh & $\begin{array}{l}\text { Average cost per case } \\
\text { (in-patient \& outpatient) }\end{array}$ \\
\hline June, 1990 & $\$ 18.60$ \\
July, 1990 & $\$ 17.64$ \\
August, 1990 & $\$ 15.06$ \\
September, 1990 & $\$ 22.07$ \\
October, 1990 & $\$ 17.99$ \\
November, 1990 & $\$ 21.64$ \\
& \\
December, 1990 & $\$ 16.60{ }^{*}$ Propofol \\
January, 1991 & $\$ 18.15$ \\
February, 1991 & $\$ 15.06$ \\
March, 1991 & $\$ 17.50$ \\
April, 1991 & $\$ 17.88$ \\
May, 1991 & $\$ 18.86$ \\
\hline
\end{tabular}

In the six-month period following the introduction of propofol the average cost per case decreased from $\$ 18.83$ to 17.34. Propofol has since gained our unrestricted use and the cost per case has not increased.

This lack of increase seems to have been the result of a decrease in the use of other anaesthetic drugs such as narcotics and inhalational agents: the cost of alfentanil decreased from $\$ 2.87$ to $\$ 1.87$ in the six months after propofol and for isoflurane, from $\$ 4.92$ to $\$ 2.80$ per case.

Our initial concerns regarding the high cost of propofol were unfounded in that the cost is offset by the lower requirement for other drugs.

C. Gerald Cooper MD FRCPC

V. Maxwell BS Pharm

Department of Anesthesia

Markham-Stouffville Hospital

Markham, Ontario L3P 7P3

\section{Tracheal intubation and cervical injury}

To the Editor:

A recent editorial by Crosby includes an apparent endorsement of the practice of inducing general anaesthesia prior to tracheal intubation in patients who may have a cervical spine injury. ${ }^{1} \mathrm{He}$ writes: "The mode of intubation should be determined by the practitioner's experience and skills and it will be the care with which the intubation is performed rather than the technique which will influence outcome." I suspect that some will interpret his commentary as a carte blanche endorsement of a "pent-sux-tube" approach for all trauma victims in need of urgent intubation. My concern is that, among trauma victims who might have a cervical spine injury, there is a subpopulation that is particularly unstable and that may be placed at high risk by this approach. This subpopulation is probably small. It would include patients with unstable upper cervical spine injuries $^{2}$ in whom atlanto-occipital extension might be particularly hazardous. Dr. Crosby may consider my suspicion as an "unsubstantiated clinical impression." However, I submit that the consequences of a cervical cord insult are such that our approach to trauma patients should take the possible presence of this subgroup into account, even if the incidence is very, very low.

To illustrate my concern, I ask how the editorialist would proceed if presented with a clinical situation, for example, two days following trauma, in which a patient with atlanto-occipital instability were presented for cervical fusion. Would it be pent-sux-tube? Or would it be an awake intubation? I submit that it would be the latter, and that in choosing the latter, he would acknowledge the high risk of lesions of this nature. Is it reasonable then to ignore the possibility of the presence of such lesions during the initial management of trauma victims?

Dr. Crosby is not alone in endorsing the practice of intubation after induction of anaesthesia. ${ }^{3,4}$ However, I have two concerns with some of the investigations that have been cited in support of the practice, including that by Dr. Crosby. ${ }^{4}$ First, they comprise populations too limited to detect the presence of a small, at-risk subset. Second, in some series patients have been submitted to a variety of intubation techniques and the selection criteria by which patients were allotted to either anaesthetized or unanaesthetized techniques is not defined. Judicious 